\title{
El servicio civil de carrera para los asesores parlamentarios mexiquenses. Análisis desde la perspectiva mexicana
}

\author{
Ma. Teresa Jaramillo Benítez \\ terejaramillob25@ hotmail.com \\ Facultad de Derecho \\ Universidad Autónoma Del Estado De México (Uaeméx) \\ Toluca, Estado de México \\ José Gabriel Góngora-Biachi \\ jg.gongora@ebc.edu.mx \\ Escuela Bancaria y Comercial \\ Métida, Yucatán, México
}

\section{RESUMEN}

Este trabajo presenta la necesidad de profesionalizar el servicio parlamentario de carrera del Congreso del Estado de México. Para profundizar en el tema, revisa los criterios de quince de los 32 estados de la República acerca de este servicio. Usando el índice de concordancia kappa, se identifica el poco acuerdo que existe entre las legislaturas locales. Tomando las mejores prácticas, este trabajo propone los puntos mínimos a considerar y el perfil de puesto de esta actividad, importante para el trabajo legislativo.

Palabras clave: servicio civil de carrera; profesionalización empleado legislativo. 


\title{
The civil career service for mexican parliamentary advisors. Analysis from the mexican perspective
}

\begin{abstract}
This paper presents the need to professionalize the career parliamentary service of the Congress of the State of Mexico. To delve into the subject, review the criteria of fifteen of the 32 states of the Republic about this service. Using the kappa concordance index, the little agreement that exists between local legislatures is identified. Taking the best practices, this work proposes the minimum points to consider and the job profile of this activity, important for legislative labor.
\end{abstract}

Keywords: career civil service, legislative employee professionalization.

Artículo recibido: 05 octubre. 2021 Aceptado para publicación: 02 noviembre 2021 Correspondencia: terejaramillob25@hotmail.com

Conflictos de Interés: Ninguna que declarar 


\section{INTRODUCCIÓN}

Este trabajo presenta una propuesta de los parámetros para el sistema de servicio civil de carrera, para los colaboradores adscritos al Poder Legislativo del Estado de México, visto como un mecanismo de fortalecimiento a la capacidad institucional de los procesos democráticos estatales, y bajo la perspectiva del derecho comparado, considerando los aportes de otros congresos locales mexicanos al tema.

Siendo una de las entidades federativas con mayor desarrollo poblacional, industrial, económico, social y político del país, llama la atención que el Estado de México no cuente con un servicio civil de carrera para los servidores públicos adscritos al Poder Legislativo. Es por el hecho de tener amplia diversidad temática y profunda complejidad en los asuntos de orden parlamentario, que la Legislatura tiende a tener altos requerimientos de información especializada, con ánimo de dar respuesta a las necesidades que la ciudadanía mexiquense reclama.

En ese contexto, surge la pregunta: ¿Qué regulación necesita el servicio civil de carrera del congreso mexiquense para fortalecer su capacidad institucional especializada en el trabajo legislativo? Para responder a ello, se realizó una investigación documental de tipo exploratorio, a la luz de la perspectiva del derecho comparado, desde los aportes de algunos congresos locales del país en la materia.

Así, el objetivo general de este trabajo es analizar la pertinencia del servicio civil de carrera como mecanismo de fortalecimiento de la función laboral de los técnicos, asesores y/o auxiliares legislativos adscritos a la legislatura, favoreciendo los procesos democráticos del estado mexiquense; en particular: 1) Establecer los aspectos a regular en un ordenamiento jurídico exprofeso de servicio civil de carrera para la legislatura mexiquense; y 2) Proponer las características y el alcance institucional mínimos que debe tener el ordenamiento jurídico del servicio civil de carrera para la Legislatura mexiquense, con base en el predominio de las experiencias y la normatividad de los congresos locales comparados. Es por ello, que este estudio es trascendente, porque analiza al servicio civil de carrera como un mecanismo institucional de fortalecimiento del perfil y desempeño de los técnicos, asesores y/o auxiliares legislativos mexiquenses.

\section{ESTRATEGIAS METODOLÓGICAS O MATERIALES Y MÉTODOS}

En el trabajo exploratorio, se analizaron las legislaciones que regulan el servicio civil de carrera en el ámbito legislativo nacional de quince de las 32 entidades federativas, 
realizando un análisis deductivo a la luz de la perspectiva del derecho comparado. Esta técnica consiste en "la comparación jurídica (...) entre discursos en torno al derecho (...) un cotejo destinado a ocuparse de los elementos de los que esos discursos derivan su alteridad... y por el contrario, aquellos otros de los que se pretenden extraer analogías y paralelismos" (Somma, 2014, pág. 78), cotejando los criterios regulados en cada uno de los ordenamientos, estableciendo las similitudes y diferencias de los mismos, para después -mediante un ejercicio interpretativo- inferir los requerimientos de un ordenamiento específico para la legislatura del Estado de México. Buscando conocer la realidad actual de este servicio, se solicitó al legislativo mexiquense mediante el Sistema de Acceso a la Información Mexiquense (SAIMEX), el número de técnicos, asesores, auxiliares o similares adscritos a la misma, para conocer su nivel de estudios y su antigüedad. Se realizó un análisis de concordancia usando el coeficiente kappa de Cohen (Siegel, \& Castellan, 2004), que es una medida estadística que ajusta el efecto del azar en la proporción de la concordancia observada para elementos cualitativos con variables categóricas. Con la información recabada, se discute para concluir con una síntesis esquemática que da pie a una propuesta de criterios a regular en materia del servicio civil de carrera, con énfasis en el perfil profesional de las figuras.

\section{RESULTADOS Y DISCUSIÓN}

\section{Referencias conceptuales del servicio civil de carrera parlamentaria en México}

El servicio civil de carrera está en expansión en el mundo entero, y ello es el reflejo del aumento de las atribuciones del Estado. La administración pública tiene cada vez más tareas, diversas y complicadas, comparadas con las que tenía en el siglo pasado. A una administración pública tradicional, cuyas funciones eran exclusivamente mantener el orden y administrar los servicios públicos, se le sustituye por una administración promotora del desarrollo económico y social, desde lo local hasta lo global, actividades que requieren de personal competente.

Aunque a nivel federal se ha implementado el Sistema Profesional de Carrera ${ }^{1}$ (SPC), no ocurre lo mismo en todas las entidades federativas. El SPC es una política pública que es clave para la profesionalización de los servidores públicos, que fomenta la eficacia de la gestión pública, buscando que se traduzca en una mejora de los servicios que ofrecen a la

\footnotetext{
${ }^{1}$ Un recuento de la evolución del servicio civil de carrera puede consultarse en Pardo (2005) y en SEDIA (2018). Ver también: Congreso de la Unión (2008).
} 
ciudadanía los poderes del Estado. Este sistema permite administrar los recursos humanos de las instituciones sujetas a la Ley de Servicio Profesional de Carrera, y garantizar su ingreso, desarrollo y permanencia en la Administración Pública Federal, a través del mérito y la igualdad de oportunidades; en un marco de transparencia y legalidad.

Desde una perspectiva académica, el servicio civil de carrera parlamentaria toma diferentes acepciones, esencialmente similares, pero con diferentes alcances; se describen a continuación, a propósito de establecer un marco conceptual que facilite la revisión y el análisis del objeto de estudio. Pardo (Pardo, 2005), comenta que el servicio civil de carrera se entiende como un conjunto de acciones sistemáticas para el ingreso, permanencia y desarrollo profesional de los servidores públicos dentro de la administración pública; Chávez (2016, pág. 167), por su parte, lo concibe como "un conjunto de reglas y procedimientos administrativos que regulan el ingreso, promoción, evaluación, remuneración y demás aspectos laborales del personal al servicio de organismos públicos"; Moyado (2006) agrega que debe ser basado en el mérito y la democracia laboral lo que regule los requisitos de incorporación al servicio público, cuidando el desarrollo del personal, la evaluación del desempeño, la permanencia, las promociones y ascensos, hasta llegar al retiro de los colaboradores. Así, con las ideas de Guerrero (2000) y Aguilar (2003), se puede sintetizar una idea clara acerca del servicio civil de carrera parlamentaria:

"conjunto de reglas y procedimientos administrativos que regulan el proceso del trabajo y su división (asignación) en una unidad administrativa. Este conjunto de reglas controla y dirige los recursos humanos de la unidad, y crea un mercado laboral cerrado que se conecta con el mercado laboral nacional a través de puntos de entrada y salida. Las reglas de entrada y salida, de permanencia y de movilidad dentro del sistema (la carrera), de asignación de las actividades y funciones de sus integrantes, y de premios y castigos a los mismos (incentivos), constituyen las características del servicio civil” (Guerrero, 2000: pp. 2); que define derechos y obligaciones, mediante una evaluación de la competencia científico-técnica y experiencia en el campo de desempeño (Aguilar, 2003). 
En sus reflexiones, Pardo (2005, pág. 608) recupera aspectos interesantes sobre el ingreso, el desarrollo profesional y la permanencia; Chávez (2016, pág. 167), adiciona el tema de la importancia de la promoción y la evaluación, considerado igual que Moyado, (2006); Aguilar (2003), agrega la relevancia del establecimiento de derechos y obligaciones, ideas que complementa Guerrero (2000, pág. 2) con reglas de entrada y salida, permanencia y movilidad, actividades, funciones, premios, incentivos y castigos.

Con la finalidad de operacionalizar para este trabajo la definición del servicio civil de carrera, éste puede ser entendido como el conjunto de reglas que norman la trayectoria laboral del funcionario, mediante la regulación de los aspectos relativos a los procesos de: 1) ingreso a la dependencia; 2) definición de las funciones, permanencia y movilidad horizontal; 3) profesionalización y actualización; 4) evaluación del desempeño y promoción (evaluación periódica, promociones verticales, premios e incentivos); 5) derechos, obligaciones, medidas correctivas y sanciones; y 6) salida (jubilación o separación del puesto). Estos procesos, aunque vastos para ser regulados en un solo ordenamiento, fueron enriquecidos en este trabajo con los hallazgos del análisis de derecho comparado sobre los ordenamientos específicos locales, buscando una propuesta para el servicio civil de carrera para el congreso mexiquense.

\subsection{El servicio civil de carrera y la función técnico-científica del parlamento}

Para identificar la necesidad de los congresos entorno al servicio civil de carrera, primero hay que entender qué es la función pública parlamentaria. En su sentido más amplio, es la creación de las normas jurídicas, la elaboración de leyes y, en lo concreto, un proceso legislativo que consiste en analizar, dictaminar, dictar y expedir las normas que rigen la conducta humana para una demarcación específica ${ }^{2}$. No obstante, el parlamento moderno no sólo interviene en la formulación de políticas públicas, sino también en la evaluación y control de éstas, como coadyuvante en la gobernabilidad y en trasparencia proactiva en la rendición de cuentas. Esta misión, requiere de una capacidad institucional basada en una estructura que permita asesoramiento altamente profesionalizado, con recursos técnicos suficientes para estos fines.

\footnotetext{
${ }^{2}$ Conceptos basados en: http://sil.gobernacion.gob.mx/Glosario/definicionpop.php?ID=192
} 
En México, los parlamentos empezaron a presentar características de profesionalización con la instauración del presidencialismo y el retiro del caudillismo, pero, sobre todo, con el advenimiento del Estado Tecnocrático en 1982 (De la Torre, 2019; Mondragón, 2014). La historia de que los legisladores cuenten con algún grado de especialización y asesoría parlamentaria es relativamente reciente. Antes se carecía de las herramientas que hacen estables y congruentes decisiones. Así, nació la necesidad de contar continuamente con asesores con conocimientos profundos, requerimiento que creció con la transdisciplinariedad de las iniciativas de ley y la cartera de asuntos de las cámaras legislativas, para recomendar, corregir o nutrir los alientos del Ejecutivo; o bien, para ejercer la función de control (Campillo, 1999).

Ser legislador es un cargo de elección popular del cual se espera que sepa que hacer y actuar por el beneficio de sus electores, pero, contradictoriamente, su profesionalización no es un requisito para el cargo. Otro tema relevante es que ningún perfil profesional, ni la experiencia de vida lo pueden especializar en las ramas del conocimiento necesaria para la naturaleza de su función multi y transdisciplinaria. Esta naturaleza amplia de su trabajo no lo inválida; por el contrario, refuerza la necesidad de asesorarse con personas que tienen conocimientos técnicos altamente especializados, mismos que no puede obtener dicho legislador en el corto período de su gestión. Es en este contexto que se requiere el servicio profesional de carrera, y destaca la necesidad de explorar en las legislaturas locales que tienen este tipo de servicio, ¿qué tipo de preparación consideran que requiere el servidor público del Poder Legislativo?, ¿cómo se adquiere la profesionalización permanente de este servidor público? La respuesta a estas preguntas, a nivel mundial, Latinoamérica y México, se enmarca en las características de los ordenamientos que regulan el servicio civil de carrera parlamentaria, coincidentes en algunas características y dispares en otras.

Aunque los órganos legislativos son entes políticos, tienen en su operación muchos componentes técnicos ${ }^{3}$. Para la calidad del trabajo legislativo, el asesoramiento parlamentario requiere de conocimiento y experiencia en distintas disciplinas (Instituto Belisario Domínguez, 2018). Así, la función pública está formada por: a) los funcionarios públicos, quienes son personas con disposición inmediata de la ley por nombramiento de autoridad competente que participa en el ejercicio de una función pública; b) los

\footnotetext{
${ }^{3}$ Es interesante revisar el informe de INEGI sobre los congresos locales (INEGI, 2020).
} 
servidores públicos, que se concibe como cualquier persona que realice cualquier actividad para el Estado, sin importar la denominación u origen; y c) los empleados, definidos como personas que realizan actividades administrativas con carácter profesional para poder brindar un servicio público a cambio de un sueldo (Jaramillo, 2005). Empero, es a partir de la existencia de asesores con formación profesional y entrenamiento en el trabajo legislativo que se incide de forma estratégica en la eficiencia y viabilidad de la gobernabilidad. Son estos asesores quienes suministran al legislador, a las comisiones y al plenario, la información necesaria y oportuna para la toma de decisiones, con productos de fácil comprensión (Escobar, 1997). Mora-Donatto (2008), reconoce la necesidad que tienen los congresos locales de:

“...contar con un apoyo técnico, profesional y permanente para nuestros legisladores. Establece, que actualmente, en los hechos, los asesores eventuales o personales... nada tienen que ver con los modernos y sofisticados staff o grupos de asesores con que cuentan la mayor parte de los parlamentos contemporáneos" (MoraDonatto, 2008, pág. 174).

Asimismo, reflexiona sobre los retos de "expertise técnica" que se necesita para asesorar en la toma de decisiones al atiene el Poder Legislativo, del cual no está exento el congreso mexiquense:

“Cada vez son más los ámbitos en los que interviene el Poder Legislativo y los problemas que tiene que enfrentar también son más complejos, específicos y técnicos, de ahí que resulta francamente increíble pretender que cada uno de los legisladores, o todos en su conjunto, sean especialistas en materias técnicas como la generación de energía nuclear, el genoma humano, la petroquímica, por sólo citar algunos” (Mora-Donatto, 2008, pág. 174).

\section{El servicio civil de carrera en las legislaturas locales, una visión comparativa desde el derecho}

El servicio civil de carrera regula la trayectoria laboral del servidor público. Esta reflexión busca establecer el alcance del marco jurídico requerido. 
Como la mayor parte de las legislaciones estatales, el ordenamiento nacional parlamentario funge como orientador de la legalidad del servicio civil de carrara de todos los congresos locales del país. El reglamento de la Cámara de Diputados del Honorable Congreso de la Unión ${ }^{4}$, establece la profesionalización de los trabajadores, favoreciendo su permanencia y promoción. En el Estatuto de la Organización Técnica y Administrativa se establece la función técnica administrativa que se realiza en el seno de la cámara de diputados $^{5}$. Particularmente, se estipulan los aspectos de reclutamiento, promoción y evaluación ${ }^{6}$; así como de nóminas y prestaciones sociales.

\section{La Ley Orgánica del Congreso General de los Estados Unidos Mexicanos, citada por}

\section{Valencia (2018, págs. 82-83) menciona como actividades de la asistencia técnica:}

1. “Comunicaciones y correspondencia, turnos y control de documentos; certificación y autentificación documental; instrumentos de identificación y diligencias relacionados con el fuero de los legisladores; registro biográfico de los integrantes de las legislaturas; y protocolo, ceremonial y relaciones públicas;

2. Servicios de la Sesión, que comprende los de: preparación y desarrollo de los trabajos del Pleno; registro y seguimiento de las iniciativas o minutas de ley o de decreto; distribución en el Pleno de los documentos sujetos a su conocimiento; apoyo a los Secretarios para verificar el quórum de asistencia; cómputo y registro de las votaciones; información y estadística de las actividades del Pleno; elaboración, registro y publicación de las actas de las sesiones; y registro de leyes y resoluciones que adopte el Pleno;

3. Servicios de las Comisiones, que comprende los de: organización y asistencia a cada una de ellas a través de su Secretario Técnico; registro de los integrantes de estas; seguimiento e información sobre el estado que guardan los asuntos turnados a Comisiones; y registro y elaboración del acta de sus reuniones;

4. Servicios del Diario de los Debates, que comprende los de: elaboración integral de la Versión Estenográfica; del Diario de los Debates; y de la Gaceta Parlamentaria;

\footnotetext{
${ }^{4}$ En el Capítulo V del Servicio de Carrera, en el artículo 283, fracción II.

${ }^{5}$ El Capítulo Séptimo del Título Segundo, de la Ley Orgánica del Congreso General de los Estados Unidos Mexicanos, "De la Organización Técnica y Administrativa".

${ }^{6}$ En el artículo 51, punto 1, inciso "a" de la Sección Tercera.
} 
5. Servicios del Archivo, que comprende los de: formación, clasificación y custodia de expedientes del Pleno y las Comisiones; y desahogo de las consultas y apoyo documental a los órganos de la Cámara y a los legisladores; y

6. Servicios de Bibliotecas, que comprende los de: acervo de libros; hemeroteca; videoteca; multimedia; museografía; e informática parlamentaria".

El Instituto Mexicano para la Competitividad (IMCO, 2018), reporta que son 24 los congresos locales que cuentan con servicio profesional de carrera (ver Ilustración 1). Para el caso del Estado de México, reporta que tienen 75 diputados, 45 de ellos elegidos por mayoría relativa $(60 \%)$ y 30 por el principio de representación proporcional (40\%), señalando que hay un diputado por cada 234,728 habitantes.

\section{Ilustración 1. Congresos locales de México que cuentan con servicio profesional de carrera}

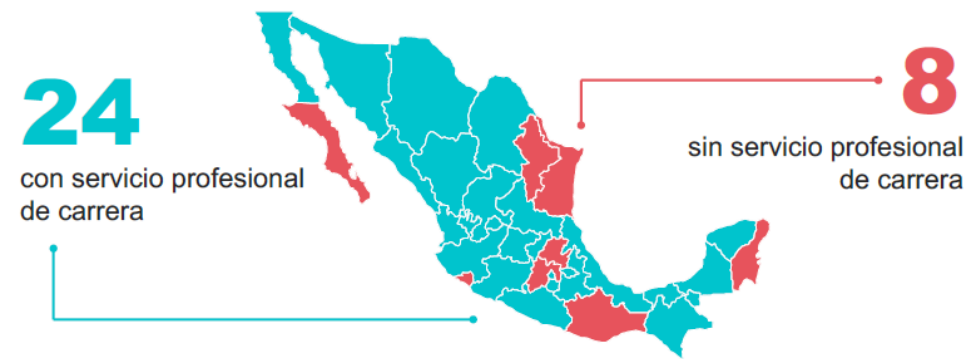

Fuente: IMCO (2018)

Utilizando la perspectiva metodológica del derecho comparado, se presentan los resultados del cotejo de los criterios regulados del servicio civil de carrera en los ordenamientos estatales específicos de los congresos locales, y de los aspectos enunciados para su regulación en las leyes orgánicas, estableciendo sus diferencias, similitudes y alcances. Las entidades fueron seleccionadas en un muestreo por conveniencia, y se muestran en la Ilustración 2. 


\section{Ilustración 2. Entidades que forman parte de la muestra}

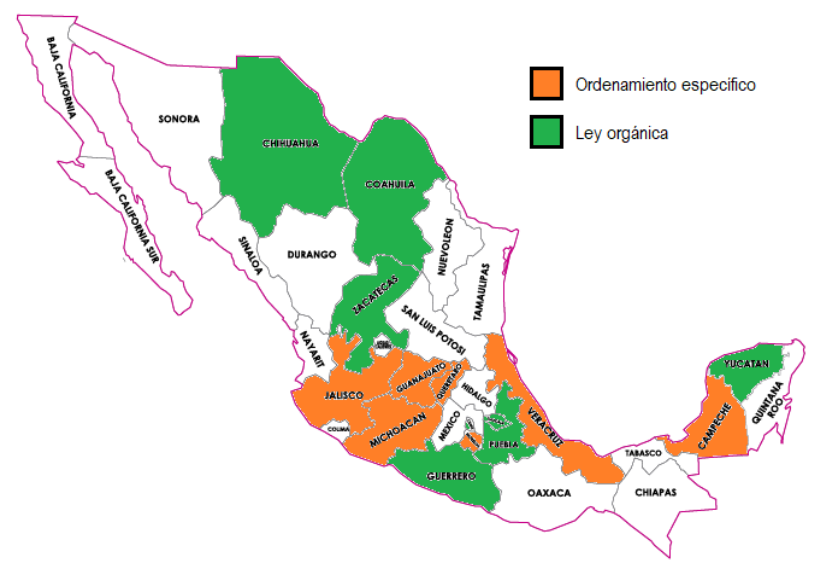

Fuente: elaboración propia

Con base en el alcance del concepto del servicio civil de carrera que norma la trayectoria laboral del funcionario -mediante la regulación de criterios de:

1) entrada;

2) permanencia y movilidad horizontal;

3) profesionalización;

4) evaluación;

5) medidas correctivas; $y$

6) salida o separación del puesto-, se presenta la comparación de 37 aspectos que abordan siete ordenamientos específicos y ocho leyes orgánicas (ver: Tabla 1).

Las leyes específicas de dos congresos (Veracruz y Querétaro) contemplan criterios relativos al ingreso, trabajo, profesionalización, evaluación del desempeño, medidas disciplinarias y separación del puesto. La primera legislación resulto ser la más completa de los siete ordenamientos revisados, con 34 de 37 aspectos regulados, mientras que la segunda Ley específica aborda 28 (siendo el cuarto ordenamiento con mayor número de aspectos normados). Los aspectos menos regulados en la segunda son los relativos a las medidas correctivas y la salida laboral. 
Tabla 1

Comparativo de los aspectos de Servicio Civil de Carrera de los ordenamientos estatales de México

\begin{tabular}{|c|c|c|c|c|c|c|c|c|c|c|c|c|c|c|c|c|}
\hline & \multirow[b]{2}{*}{\begin{tabular}{lr} 
Rubro & del \\
personal & de \\
servicio civil de & carrera \\
contemplado en \\
\multicolumn{2}{l}{ los ordenamientos }
\end{tabular}} & \multicolumn{7}{|c|}{ Ordenamientos específicos } & \multicolumn{8}{|c|}{ Leyes orgánicas } \\
\hline & & 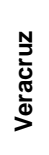 & 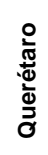 & 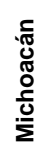 & 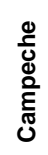 & 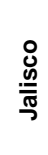 & $\begin{array}{l}0 \\
\frac{0}{\pi} \\
\stackrel{3}{\pi} \\
\frac{\pi}{\pi} \\
\frac{\pi}{\pi} \\
0 \\
0\end{array}$ & $\begin{array}{l}\frac{0}{0} \\
\frac{0}{20} \\
\frac{0}{2}\end{array}$ & 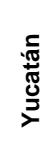 & 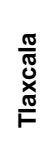 & $\frac{\pi}{0}$ & $\begin{array}{l}\stackrel{g}{0} \\
\mathbb{d} \\
\mathbb{8} \\
\mathbb{N} \\
\mathbb{N}\end{array}$ & 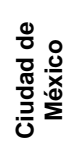 & 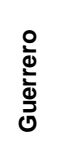 & $\begin{array}{l}\frac{\pi}{3} \\
\frac{\pi}{\pi} \\
0\end{array}$ & 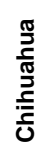 \\
\hline & \multicolumn{16}{|l|}{ Entrada } \\
\hline 1 & Ingreso & घ & $\mathbf{E}$ & [ & [ & [ & [ & 口 & घ & [ & & & [ & & & \\
\hline 2 & Vacantes & $\bar{\square}$ & $\bar{\square}$ & $\mathbf{E}$ & $\bar{\square}$ & $\bar{\square}$ & $\bar{\square}$ & $\overline{\mathbf{n}}$ & & & & & & & & \\
\hline 3 & Selección & प & 口 & a & [ & ( & [ & [ & [ & $\mathbf{\square}$ & [ & $\mathbf{\square}$ & & & & \\
\hline 4 & Reclutamiento & $\bar{\square}$ & $\bar{\square}$ & $\mathbf{\square}$ & $\bar{\square}$ & $\bar{\square}$ & $\mathbf{\square}$ & $\mathbf{\square}$ & & & & & & & & \\
\hline 5 & Perfil de puestos & $\bar{\square}$ & $\bar{\square}$ & a & $\bar{\square}$ & $\bar{\square}$ & $\mathbf{E}$ & $\bar{\square}$ & [ & [ & [ & [ & [ & & & \\
\hline \multirow[t]{2}{*}{6} & Inducción & $\bar{\square}$ & & $\bar{\square}$ & $\bar{\square}$ & & $\bar{\square}$ & & & & & & & & & \\
\hline & \multicolumn{16}{|l|}{$\begin{array}{l}\text { Permanencia y } \\
\text { Movilidad } \\
\text { horizontal }\end{array}$} \\
\hline 7 & Permanencia & E & 口 & [ & [ & & [ & E & [ & [ & $\mathbf{\square}$ & E & [ & $\overline{\mathbf{n}}$ & & $\bar{\square}$ \\
\hline 8 & Designaciones & $\bar{\square}$ & & $\bar{\square}$ & [ & & & a & & & & & & & & \\
\hline 9 & Nombramientos & $\bar{\square}$ & 口 & a & $\bar{\square}$ & [ & [ & $\bar{\square}$ & & & & & & & & \\
\hline 10 & $\begin{array}{l}\text { Puesto o estructura } \\
\text { ocupacional o } \\
\text { niveles jerárquicos }\end{array}$ & घ & घ & घ & घ & घ & घ & घ & & घ & घ & घ & घ & & & \\
\hline 11 & Plaza & [ & [ & & [ & E & & & & & & & & & & \\
\hline 12 & $\begin{array}{l}\text { Movilidad } \\
\text { horizontal }\end{array}$ & घ & घ & च & & [ & & घ & & & & & & & & \\
\hline 13 & Intercambios & & & [ & & & & & & & & & & & & \\
\hline \multirow[t]{2}{*}{14} & Permutas & $\mathbf{E}$ & 口 & & & & & & & & & & & & & \\
\hline & \multicolumn{16}{|l|}{ Profesionalización } \\
\hline 15 & $\begin{array}{l}\text { Formación } \\
\text { profesional }\end{array}$ & घ & घ & घ & घ & घ & घ & घ & & & & & & घ & घ & घ \\
\hline 16 & Especialización & [ & 口 & E & & & E & [ & & & & & E & [ & & \\
\hline 17 & Actualización & $\bar{\square}$ & $\bar{\square}$ & $\mathbf{\square}$ & & E & $\bar{\square}$ & $\overline{\mathbf{a}}$ & $\bar{\square}$ & $\bar{\square}$ & 口 & $\mathbf{E}$ & $\bar{\square}$ & $\bar{E}$ & & \\
\hline 18 & Certificaciones & $\overline{\mathbf{a}}$ & & $\overline{\mathbf{a}}$ & & $\bar{\square}$ & & & & & & & & & & \\
\hline \multirow[t]{2}{*}{19} & Capacitación & E & E & [ & [ & E & E & $\overline{\mathbf{n}}$ & $\mathbf{\square}$ & [ & 口 & $\mathbf{E}$ & & $\overline{\mathbf{n}}$ & & $\mathbf{\square}$ \\
\hline & \multicolumn{16}{|l|}{$\begin{array}{l}\text { Evaluación y } \\
\text { Promoción }\end{array}$} \\
\hline 20 & $\begin{array}{l}\text { Evaluación del } \\
\text { desempeño }\end{array}$ & घ & घ & घ & घ & घ & घ & घ & घ & & & & & & & \\
\hline 21 & Promociones & E & 口 & 口 & E & E & E & $\mathbf{E}$ & $\mathbf{\square}$ & $\overline{\mathbf{n}}$ & E & 口 & $\mathbf{\square}$ & 口 & & \\
\hline 22 & Asensos & [ & 口 & & [ & [ & [ & [ & $\mathbf{E}$ & 口 & $\mathbf{\square}$ & [ & [ & - & & \\
\hline 23 & Incentivos & $\bar{\square}$ & $\bar{\square}$ & a & $\bar{\square}$ & & $\bar{\square}$ & $\bar{\square}$ & & & & & & & & \\
\hline 24 & Estímulos & E & 口 & $\mathbf{E}$ & 口 & & [ & $\mathbf{E}$ & [ & 口 & [ & $\mathbf{E}$ & & & & \\
\hline 25 & Reconocimientos & $\overline{\mathbf{n}}$ & $\bar{\square}$ & $\bar{\square}$ & $\bar{\square}$ & & & $\bar{\square}$ & & & & & & & & \\
\hline \multirow[t]{2}{*}{26} & Beneficios & & 口 & & & $\mathbf{E}$ & E & & & & & & & & & \\
\hline & \multicolumn{16}{|l|}{$\begin{array}{l}\text { Medidas } \\
\text { correctivas }\end{array}$} \\
\hline 27 & Derechos & प & 口 & a & [ & a & [ & [ & & & & & & & & \\
\hline 28 & Obligaciones & $\bar{\square}$ & $\bar{\square}$ & a & $\bar{\square}$ & a & $\bar{\square}$ & a & & & & & E & & & \\
\hline 29 & Infracciones & $\bar{\square}$ & & & $\bar{\square}$ & & & & & & & & & & & \\
\hline 30 & Sanciones & E & & $\bar{\square}$ & E & [ & & E & & & & & & & & \\
\hline 31 & Causas de baja & $\mathbf{a}$ & & $\bar{\square}$ & $\mathbf{\square}$ & $\overline{\mathbf{a}}$ & $\mathbf{\square}$ & $\bar{\square}$ & & & & & & & & \\
\hline \multirow[t]{2}{*}{32} & $\begin{array}{l}\text { Recursos de } \\
\text { inconformidad o } \\
\text { revocación }\end{array}$ & घ & घ & घ & घ & a & घ & घ & & & & & & & & \\
\hline & \multicolumn{16}{|l|}{ Salida } \\
\hline 33 & Permisos & & 口 & & [ & & [ & 口 & & & & & & & & \\
\hline 34 & Licencias & E & $\bar{\square}$ & 口 & $\bar{\square}$ & 口 & $\overline{\mathbf{n}}$ & $\overline{\mathbf{a}}$ & & & & & & & & \\
\hline 35 & Reincorporación & 口 & 口 & [ & & [ & & [ & & & & & & & & \\
\hline 36 & $\begin{array}{l}\text { Separación del } \\
\text { puesto }\end{array}$ & a & घ & a & घ & ש & [ & ש & घ & & & & & & & \\
\hline 37 & Reingreso & $\bar{\square}$ & & & & E & & & & & & & & & & \\
\hline & Total & 34 & 29 & 30 & 28 & 26 & 26 & 29 & 11 & 10 & 9 & 9 & 9 & 7 & 1 & 3 \\
\hline
\end{tabular}




\section{La Tabla 1 fue elaborada con base en las diferentes legislaciones locales ${ }^{7}$}

En cuanto al congreso de Michoacán, segundo ordenamiento con mayor número de aspectos regulados (con 30 de 37 aspectos), se observó que en los procesos de salida laboral, evaluación y permanencia y movilidad horizontal no se consideraron dos elementos. Campeche y Jalisco, básicamente no consideraron aspectos relativos a la permanencia y movilidad horizontal, y a la salida laboral.

En cuanto a los aspectos enunciados para su regulación en las leyes orgánicas, se observó que abordaron como máximo doce de los 37 considerados en los por la totalidad de los ordenamientos, siendo la Ley del congreso de Yucatán la que mayor número de elementos expresa (12), mientras que los ordenamientos de los congresos de Coahuila y Chihuahua sólo enuncian tres o menos rubros para su regulación.

Todos los ordenamientos tuvieron diferentes alcances en el campo de acción de su competencia y objeto de regulación, siendo el más robusto y especifico el ordenamiento del congreso del estado de Veracruz, seguido del congreso de Michoacán y Morelos, después el de Querétaro, subsiguientemente el congreso de Campeche, seguido de Guanajuato y Jalisco, detrás el Congreso de Yucatán y Tlaxcala, posteriormente los congresos de los estados de Puebla, Ciudad de México y Zacatecas, y por último, los congresos de los estados de Guerrero, Coahuila y Chihuahua.

\footnotetext{
${ }^{7}$ Ley Orgánica del Congreso de la Ciudad de México. 2019.

Ley Orgánica del Poder Legislativo del Estado Libre y Soberano de Puebla. 2020

Ley Orgánica del Poder Legislativo del Estado de Tlaxcala. 2018.

Ley Orgánica del Poder Legislativo del Estado de Zacatecas. 2020.

Ley Orgánica del Poder Legislativo del Estado de San Luis Potosí. 2008.

Ley Orgánica del Poder Legislativo del Estado de Nayarit. 2020.

Ley Orgánica del Congreso del Estado Independiente Libre y Soberano de Coahuila de Zaragoza. 2020.

Ley Orgánica del Poder Legislativo del Estado de Chihuahua. 2016.

Ley Orgánica del Poder Legislativo del Estado Libre y Soberano de México. 1995.

Ley Orgánica del Poder Legislativo del Estado Libre y Soberano de México. 2019

Estatuto del Servicio Civil de Carrera de los Servidores Públicos del Poder Legislativo. Guanajuato. México. 2012

Estatuto que rige el Servicio Civil de Carrera en el Congreso del Estado de Morelos. 2003.

Ley del Sistema de Servicio Profesional de Carrera del Poder Legislativo del Estado de Querétaro. 2008.

Ley del Servicio Profesional de Carrera del Congreso del Estado de Veracruz. 2004.

Reglamento del Poder Legislativo del Estado Libre y Soberano de México. 1995.

Ley Orgánica del Poder Legislativo del Estado de Guerrero. 2020.

Reglamento del Servicio Parlamentario de Carrera del Congreso del Estado de Michoacán de Ocampo. 2012.

Reglamento del Servicio Civil de Carrera del Poder Legislativo del Estado de Campeche. 2018.
} 
A la luz de los quince procesos normados en los ordenamientos específicos y las leyes orgánicas de los congresos estatales, se analizaron los ordenamientos básicos del Poder Legislativo del Estado de México en cuanto a materia de regulación laboral, a propósito de que los hallazgos cimentaran la propuesta de implementación del servicio civil de carrera.

En cuanto a criterios regulados, mostrados en la Tabla 1, los ordenamientos tuvieron las siguientes similitudes: 1) Entrada (ingreso, vacantes, selección, reclutamiento, perfil de puestos e inducción); 2) Permanencia y movilidad horizontal (permanencia, designaciones, nombramientos, puesto o estructura ocupacional o niveles jerárquicos, plaza y movilidad horizontal); 3) Profesionalización (formación profesional, especialización, actualización, certificaciones y capacitación); 4) Evaluación (evaluación del desempeño, promociones, asensos, incentivos, estímulos y reconocimientos; 5) Medidas correctivas (derechos, obligaciones, causas de baja y recursos de inconformidad o revocación); y 6) Salida (licencias, reincorporación y separación del puesto). Los rubros que destacaron por su coincidencia en la ausencia mayoritaria son: beneficio, intercambios, permutas, reingreso, permisos, infracciones y sanciones.

Para medir la concordancia entre las entidades federativas se utilizó el estadístico kappa $(\mathrm{K})$, que mide la razón de la proporción de veces que los evaluadores estuvieron de acuerdo con la proporción máxima de veces que podrían concordar (ambos términos corregidos para acuerdo aleatorio) usando el método que proponen Siegel y Castellan (2007). Se determinó también si el valor observado fue más grande que el valor que se esperaría al azar. Así mismo, mediante valores obtenidos en el cálculo del estadístico kappa, se determinó la proporción de concordancia en la evaluación de cada ítem entre las entidades federativas participantes. Los resultados se muestran en la ¡Error! La autoreferencia al marcador no es válida.

Analizando la concordancia de los índices, se observa que el índice es teórico de concordancia es de $\mathrm{P}(\mathrm{E})=0.502$, mientras que en la evaluación concuerdan $\mathrm{P}(\mathrm{A})=$ 0.02703, siendo el índice negativo $(K=-0.95)$, y estadísticamente no significativo. 


\section{Tabla 2}

\section{Índice de concordancia entre las entidades}

\begin{tabular}{|c|c|c|c|}
\hline & Rubro del personal de servicio civil de carrera & $\mathbf{N}$ & Concordancia \\
\hline & Entrada & & \\
\hline 1 & Ingreso & 10 & 0.524 \\
\hline 2 & Vacantes & 7 & 0.467 \\
\hline 3 & Selección & 11 & 0.581 \\
\hline 4 & Reclutamiento & 7 & 0.467 \\
\hline 5 & Perfil de puestos & 12 & 0.657 \\
\hline \multirow[t]{2}{*}{6} & Inducción & 4 & 0.581 \\
\hline & Permanencia y Movilidad horizontal & & \\
\hline 7 & Permanencia & 13 & 0.752 \\
\hline 8 & Designaciones & 4 & 0.581 \\
\hline 9 & Nombramientos & 7 & 0.467 \\
\hline 10 & Puesto o estructura ocupacional o niveles jerárquicos & 11 & 0.581 \\
\hline 11 & Plaza & 4 & 0.581 \\
\hline 12 & Movilidad horizontal & 5 & 0.524 \\
\hline 13 & Intercambios & 1 & 0.867 \\
\hline \multirow[t]{2}{*}{14} & Permutas & 2 & 0.752 \\
\hline & Profesionalización & & \\
\hline 15 & Formación profesional & 10 & 0.524 \\
\hline 16 & Especialización & 7 & 0.467 \\
\hline 17 & Actualización & 12 & 0.657 \\
\hline 18 & Certificaciones & 3 & 0.657 \\
\hline \multirow[t]{2}{*}{19} & Capacitación & 13 & 0.752 \\
\hline & Evaluación y Promoción & & \\
\hline 20 & Evaluación del desempeño & 8 & 0.467 \\
\hline 21 & Promociones & 13 & 0.752 \\
\hline 22 & Asensos & 12 & 0.657 \\
\hline 23 & Incentivos & 6 & 0.486 \\
\hline 24 & Estímulos & 10 & 0.524 \\
\hline 25 & Reconocimientos & 5 & 0.524 \\
\hline \multirow[t]{2}{*}{26} & Beneficios & 3 & 0.657 \\
\hline & Medidas correctivas & & \\
\hline 27 & Derechos & 7 & 0.467 \\
\hline 28 & Obligaciones & 8 & 0.467 \\
\hline 29 & Infracciones & 2 & 0.752 \\
\hline 30 & Sanciones & 5 & 0.524 \\
\hline 31 & Causas de baja & 6 & 0.486 \\
\hline \multirow[t]{2}{*}{32} & Recursos de inconformidad o revocación & 7 & 0.467 \\
\hline & Salida & & \\
\hline 33 & Permisos & 4 & 0.581 \\
\hline 34 & Licencias & 7 & 0.467 \\
\hline 35 & Reincorporación & 5 & 0.524 \\
\hline 36 & Separación del puesto & 8 & 0.467 \\
\hline \multirow[t]{3}{*}{37} & Reingreso & 2 & 0.752 \\
\hline & Total & 7 & 0.580 \\
\hline & $\begin{array}{l}\text { CONCUERDAN AL AZAR P(E)=0.502 } \\
\text { LOS EVALUADORES CONCUERDAN } P(A)=0.02703 \\
K=-0.95 \text { NO SIGNIFICATIVO }\end{array}$ & & \\
\hline
\end{tabular}

Fuente: elaboración propia

De lo anterior, se infiere que el servicio civil de carrera parlamentaria tiene poca concordancia entre los ítems estudiados de las legislaciones locales, siendo que es considerado como mecanismo de fortalecimiento de capacidad institucional de los congresos a propósito del mejoramiento democrático. Esto sugiere la necesidad de armonizarlos para que incluyan los mismos aspectos en su legislación, y éstas sean más robustas. 


\section{La ausencia del servicio civil de carrera del parlamento mexiquense}

El Estado de México es una de las Entidades Federativas con mayor número de desarrollo poblacional, industrial, económico, social y político, es de esperarse que sus instituciones gubernamentales estén a la altura de los reclamos de su ciudadanía, toda vez que la Legislatura del Estado de México representa la voluntad del pueblo mexiquense.

En el Congreso mexiquense participan diputados (servidores públicos de elección popular), funcionarios públicos (personas con disposición inmediata de la ley por nombramiento de autoridad competente, que participa en el ejercicio de una función pública) y empleados (personas que realizan actividades administrativas, con carácter profesional, para poder brindar un servicio público a cambio de un sueldo) (Jaramillo, 2005). Las dependencias que integran el Poder Legislativo del estado de México son: el Órgano Superior de Fiscalización, la Secretaria de Asuntos Parlamentarios, la Contraloría del Poder Legislativo, la Secretaria de Administración y Finanzas, el Instituto de Estudios Legislativos, la Unidad de Información y la Biblioteca "Dr. José María Luis Mora" (Reyes Pastrana, 2020).

Para inicios de 2021, el Poder Legislativo estaba integrado por 2,167 servidores públicos, de los cuales 37 eran técnicos, asesores y/o auxiliares legislativos o similares; el $72.9 \%$ eran hombres y el $27.1 \%$ mujeres; el $91.4 \%$ tenían edad entre 20 y 55 años, y el $8.6 \%$ de 56 años en adelante. Tienen encomiendas disciplinares, como las de investigación, asesoría, administrativas y financieras.

El 100\% de los técnicos, asesores y/o auxiliares legislativos o similares son empleados de confianza, con tres o menos años de antigüedad, y no cuentan con el servicio civil de carrera que les ofrece la posibilidad de desarrollar una carrera en el servicio público. El $89.1 \%$ cuenta con formación de licenciatura o estudios de posgrado y conforman un grupo interdisciplinario (catorce asesores con licenciaturas diferentes), lo que muestra un capital intelectual con la necesidad y pertinencia de la profesionalización continua y permanente. El 39.2\% cuenta con formación en derecho y el $60.8 \%$ restante en ciencias políticas, administración, contaduría, ingeniería química, comercio internacional y aduanas, economía, comunicación, historia, relaciones Internacionales, turismo, sociología, negocios internacionales y medicina. 


\subsection{La importancia del servicio civil de carrera para el asesor parlamentario mexiquense}

Es de resaltar la necesidad que tiene la actividad legisladora de contar con apoyo técnico, profesional, permanente, objetivo y apartidista, como una forma de diversificar las fuentes de información en el ámbito de los asuntos públicos, ya que esto representará un factor de fortalecimiento democrático para los legisladores.

En una sociedad que crece numéricamente, y cada vez más dinámica, son mayores los ámbitos en los que interviene el Poder Legislativo mexiquense. Los problemas que tiene que enfrentar también son más complejos, específicos y técnicos, por lo que resulta absurdo pretender que los legisladores sean especialistas en temas técnicos como el análisis de la cuenta pública, el presupuesto de egresos, la seguridad pública, la educación, por sólo citar algunos. Y aunque el Poder Legislativo no está solo en las tareas de proporcionar información, ya que hay organizaciones de la sociedad civil que impulsan la transparencia y la rendición de cuentas de los poderes legislativos, monitoreando su trabajo y actividad legisladora, es imprescindible la presencia de asesores parlamentarios, capacitados científicamente para desarrollar actividades de apoyo legislativo.

Las funciones que realizan los técnicos, asesores y/o auxiliares o similares para dar soporte a la función legislativa mexiquense tienen la fortaleza de la interdisciplinaridad, pero requiere un máximo de especialización, lo cual toma mayor importancia si se considera que en su totalidad son servidores de confianza lo que indica que su nivel de responsabilidad es similar a la de un mando medio (jefe de departamento) y más cercana a la de un mando alto (directivo) que a la de un operativo. Pero, la permanencia en la actividad no está regulada, perdiéndose la experiencia. Los técnicos, asesores y/o auxiliares o similares tienen tres o menos años de antigüedad laborando para la cámara, mostrando la rotación de servidores públicos que se da con cada cambio de Legislatura. Y, aunque la Ley Orgánica del Poder Legislativo confiere la atribución de impulsar programas de profesionalización y actualización en derecho parlamentario y prácticas legislativas para el personal, y el Reglamento del Poder Legislativo establece la atribución de aplicar programas de incentivos, calificación de méritos y evaluación del rendimiento para los servidores públicos legislativos, ambos ordenamientos no consideran la estabilidad y la continuidad laboral, ni la profesionalización interdisciplinaria. 
El Congreso mexiquense, por conducto de la Secretaría de Asuntos Parlamentarios ${ }^{8}$, prevé que las comisiones y comités contarán con apoyo técnico que sea pertinente para la formulación de proyectos de dictamen o de informe. Las actividades relativas incluyen asesoramiento de profesionales expertos que brinden soporte en materia de fondo, como son: El procedimiento parlamentario, la técnica legislativa y control político, que implica estudios, informes, minutas, consultas, apoyo logístico, documentos de base para los discursos, entre otros servicios, insumos que requieren de brindarse en forma analítica, imparcial, objetiva y oportuna.

Es en este sentido deriva la importancia para desarrollar la capacidad institucional en materia de asesoría técnico-científica para conocer a profundidad los temas que son de su competencia y así poder contar con vastas y diversas alternativas o escenarios antes de la toma de decisiones, así como una amplia información situacional y diagnostica detallada, tener datos suficientes y objetivos que le den sentido a los análisis, como también tener a su alcance todo tipo de documentos y estudios que den fundamento y soporte a sus reflexiones, análisis, juicios, opiniones y votos y prevenir la parcialidad, la corrupción, la impunidad, el nepotismo, el autoritarismo y el clientelismo político.

Analizando la Ley Orgánica del Poder Legislativo del Estado Libre y Soberano de México (1995), en los siguientes artículos (ver Tabla 3) se considera el apoyo y la asesoría técnica como una función parlamentaria.

Los aspectos de la vida laboral de los servidores públicos del Poder Legislativo que contempla (no regula ni establece criterios normativos) solo observa lo relativo a los nombramientos, el catálogo de puestos, parte del proceso de permanencia y movilidad horizontal; y la actualización como elemento de la profesionalización. De los seis procesos y los 37 aspectos que norman la mayoría de los ordenamientos de los congresos locales, la Ley orgánica del Poder Legislativo del Estado de México considera sólo cuatro aspectos.

Es importante abundar que en el Reglamento del Poder Legislativo del Estado Libre y Soberano de México (1995), se establece la función de apoyo y asesoría técnica para la función legislativa en la figura de los secretarios técnicos, que son parte del grupo de asesores con los que opera el parlamento mexiquense.

\footnotetext{
${ }^{8}$ Párrafo segundo del artículo 70 del Capítulo VI. De las Comisiones y Comités de la Ley Orgánica del Poder Legislativo del Estado Libre y Soberano de México (1995)
} 
Específicamente, sobre el proceso de entrada, se enuncia la expedición de nombramientos; la evaluación del rendimiento y la posibilidad de establecer programas de incentivos y estímulos con base en la calificación de méritos; y remuneraciones acordes al desempeño. En materia de medidas correctivas, se enuncian procedimientos administrativos y sanciones; y, por último, se observó, el establecimiento de la emisión de licencias temporales o absolutas, así como el tratamiento a las renuncias.

En conjunto, se observó que la Ley Orgánica y el Reglamento del Congreso del Estado de México, abordan los seis procesos que regulan los congresos locales para la trayectoria laboral del servicio civil de carrera. Son once los aspectos considerados, de los 37 regulados. Sin embargo, existe un vacío orgánico que es necesario llenar mediante un replanteamiento laboral que garantice, tanto la pericia de los técnicos, asesores y/o auxiliares legislativos o similares, como el reclutamiento del personal idóneo para ocupar estos cargos y tercero, un sistema que motive e incentive los dos puntos anteriores, así como la permanencia de estos.

\section{Tabla 3.}

\section{Mandatos de ley relacionados con la asesoría parlamentaria}

\begin{tabular}{l} 
La Junta de Coordinación Política podrá constituir la Mesa Técnica, como un órgano de \\
asesoría y opinión, para facilitar los trabajos de estudio de las iniciativas y de los \\
asuntos turnados a las Comisiones o Comités, que le sean encomendados por la Junta de \\
Coordinación Política. Artículo 96 Ter. \\
La Secretaría de Asuntos Parlamentarios; el Secretario Técnico de la Junta de \\
Coordinación Política; el Coordinador de Enlace Parlamentario o su equivalente que será \\
designado por la Junta de Coordinación Política; los Coordinadores de Asesores de los \\
diferentes grupos parlamentarios o sus equivalentes. Artículo 70. \\
\hline La Secretaría de Asuntos Parlamentarios, las comisiones y comités permanentes \\
contarán con el apoyo técnico de carácter jurídico que sea pertinente para la \\
formulación de proyectos de dictamen o de informes, así como para el levantamiento \\
y registro de las actas de sus reuniones... Artículo 70 \\
\hline Son atribuciones del presidente de la Junta de Coordinación Política ... Formular a la Junta \\
de Coordinación Política las propuestas que deban ser sometidas a la Asamblea para \\
nombrar a los servidores públicos del Poder Legislativo cuya designación esté \\
reservada a ésta, o aquellas que la Ley establezca. Artículo 65. \\
Impulsar programas de profesionalización y actualización en derecho parlamentario y \\
prácticas legislativas. Artículo 76 B. \\
\hline Son atribuciones de la Junta de Coordinación Política ... Proponer a la Asamblea, a más \\
tardar en el mes de noviembre, el Tabulador de remuneraciones de los servidores públicos \\
del Poder Legislativo que deberá seguir durante el siguiente ejercicio fiscal, de conformidad \\
con el Catálogo General de Puestos y agregado a los criterios establecidos por la Ley. \\
Artículo 62 .
\end{tabular}

Fuente: Elaboración propia. Ley Orgánica del Poder Legislativo del Estado Libre y

Soberano de México. 1995. 


\subsection{Propuesta de criterios para un ordenamiento de servicio civil de carrera de la Legislatura mexiquense}

El objeto de este ordenamiento es la regulación de la trayectoria laboral del servidor público parlamentario mediante el mecanismo del Servicio civil de Carrera. Es deseable que contemple criterios normativos para toda la trayectoria del personal de asesoría del Poder Legislativo, desde su postulación para ingreso, hasta posibles inconformidades debido a su separación, pasando por las fases sustantivas e intermedias de profesionalización, movilidad, promoción, incentivos y permanencia.

Con base en las acepciones analizadas, se propone la siguiente definición de Servicio Civil de Carrera: Conjunto de normas que regulan los procedimientos de entrada o inserción; permanencia y movilidad; profesionalización; promoción; medidas correctivas; y salida o separación del puesto, mediante una evaluación de la competencia científico-técnica en el campo de desempeño de los recursos humanos que integran la administración pública parlamentaria, con el propósito de elevar la profundidad y especialidad del trabajo legislativo y, en consecuencia, de los procesos democráticos locales. El propósito de este conjunto de normas es el de fortalecer el trabajo y la toma de decisiones propias de las obligaciones y responsabilidades de la función pública, así como los procesos democráticos que encabeza el Poder legislativo, mediante la regulación del servicio civil de carrera para la profesionalización y permanencia de los funcionarios públicos especializados que dan asesoría parlamentaria.

Con base en las similitudes derivadas del análisis comparativo de los ordenamientos de los congresos locales, se presenta en la Tabla 4 el resumen de los criterios normativos que debe contemplar una legislación en materia de servicio de carrera parlamentaria. Los criterios normativos referidos muestran las dimensiones de entrada, desarrollo profesional y permanencia para el asesor parlamentario, personal del servicio civil de carrera. Se incluyen aspectos tales como la permanencia en el empleo y la profesionalización del colaborador, soporte técnico y especializado de los legisladores. 
Tabla 4.

Criterios normativos de la legislación del servicio de carrera parlamentaria

\begin{tabular}{|c|c|c|c|c|c|}
\hline \multicolumn{6}{|c|}{ Capítulos } \\
\hline I & II & III & IV & $\mathbf{V}$ & VI \\
\hline Entrada & $\begin{array}{c}\text { Permanencia y } \\
\text { movilidad } \\
\text { horizontal }\end{array}$ & Profesionalización & $\begin{array}{l}\text { Evaluación y } \\
\text { Promoción }\end{array}$ & $\begin{array}{c}\text { Medidas } \\
\text { correctivas }\end{array}$ & Salida \\
\hline 1. Ingreso & 1. Permanencia & $\begin{array}{l}\text { 1. Formación } \\
\text { profesional }\end{array}$ & $\begin{array}{l}\text { 1. Evaluación del } \\
\text { desempeño }\end{array}$ & 1. Derechos & 1. Permisos \\
\hline 2. Vacantes & 2. Designación & 2. Especialización & 2. Promociones & $\begin{array}{l}2 . \\
\text { Obligaciones }\end{array}$ & 2. Licencias \\
\hline 3. Selección & 3. Nombramientos & 3. Actualización & 3. Asensos & 3. Infracciones & $\begin{array}{l}3 . \\
\text { Reincorporación }\end{array}$ \\
\hline 4. Reclutamiento & $\begin{array}{l}\text { 4. Puesto o } \\
\text { estructura } \\
\text { ocupacional o } \\
\text { niveles } \\
\text { jerárquicos }\end{array}$ & 4. Certificaciones & 4. Incentivos & 4. Sanciones & $\begin{array}{l}\text { 4. Separación } \\
\text { del puesto }\end{array}$ \\
\hline $\begin{array}{l}\text { 5. Perfil de } \\
\text { puesto }\end{array}$ & 5. Plaza & 5. Capacitación & 5. Estímulos & $\begin{array}{l}\text { 5. Causas de } \\
\text { baja }\end{array}$ & 5. Reingresos \\
\hline 6. Inducción & $\begin{array}{l}\text { 6. Movilidad } \\
\text { horizontal }\end{array}$ & & $\begin{array}{l}6 . \\
\text { Reconocimientos }\end{array}$ & $\begin{array}{l}\text { 6. Recursos de } \\
\text { inconformidad } \\
\text { o revocación }\end{array}$ & \\
\hline & 7. Intercambios & & 7. Beneficios & & \\
\hline & 8. Permutas & & & & \\
\hline
\end{tabular}

Fuente: Elaboración propia

\section{Tabla 5.}

\section{Perfil de puesto para el asesor parlamentario}

\section{Asesor Parlamentario}

\section{RESPONSABILIDAD}

Asesorar y suministrar información sobre temas específicos para facilitar la toma de las decisiones; sistematizar antecedentes y preparar argumentos para afrontar la deliberación en la Comisión y el debate en el Pleno; redactar proyectos; colaborar en la formulación de dictámenes, y proveer herramientas que permitan el desarrollo del trabajo parlamentario de los legisladores.

\section{FUNCIONES ESPECÍFICAS}

\section{Asesorar}

Proyectar Iniciativas de Ley

Diseñar y ejecutar procedimiento parlamentario

Aplicar técnica legislativa

Ejecutar control político

Realizar estudios específicos

Formular informes

Elaborar minutas

Hacer consultas

Dar apoyo logístico

Integrar documentos

Desarrollar discursos

\section{ESCOLARIDAD NECESARIA}

\section{CARRERAS AFINES PARA EL DESEMPEÑO DEL PUESTO:}

Licenciado en Derecho, Ciencias Políticas, Administración Pública y Ciencias afines a las Sociales.

Dependiendo de la Comisión y/o Comité al que se adscriba, será la licenciatura con especialidad, o bien maestría.

\section{HABILIDADES Y ACTITUDES PARA EL PUESTO}

\begin{tabular}{l|l}
\hline Habilidades Actitudes & Sistematización \\
Compresión de la información & Objetividad \\
Expresión verbal asertiva & Síntesis \\
Juicio crítico & Iniciativa \\
Negociación & Evaluación \\
Análisis y redacción & Disponibilidad \\
Imparcialidad & \\
\hline
\end{tabular}

Fuente: elaboración propia 
También, es de interés esbozar el perfil de puesto del asesor parlamentario. La Tabla 5 muestra esta propuesta. Este diseño de puesto, aunque genérico, permite ver la razón de ser del asesor parlamentario, muestra la amplitud y especificidad de sus funciones, así como las características aptitudinales y actitudinales con las que cuenta para hacer efectiva su labor en pos de la objetividad del trabajo parlamentario.

\section{CONCLUSIÓN O CONSIDERACIONES FINALES}

El objetivo general de este trabajo fue analizar la pertinencia del servicio civil de carrera como mecanismo de fortalecimiento de la función laboral de los técnicos, asesores y/o auxiliares legislativos adscritos a la legislatura, favoreciendo los procesos democráticos del estado mexiquense. Para ello, se compararon los ordenamientos de quince legislaturas locales, encontrando escasa coincidencia al aplica el índice de concordancia del estadístico kappa, para medir la razón de la proporción de veces que los evaluadores estuvieron de acuerdo con la proporción máxima de veces que podrían concordar.

Debido a sus funciones y actividades, es de suma importancia analizar y esbozar el objetivo y perfil profesional del asesor parlamentario, en el marco del servicio civil de carrera, para establecer la permanencia y elevar la competencia de los colaboradores que forman parte de este servicio en el Poder Legislativo del Estado de México. Esto contribuye a sentar las bases de su posible implementación en el corto, mediano o largo plazo de este servicio y, con ello, se logrará capitalizar la experticia institucional, profesionalizando a los colaboradores del poder legislativo y elevando la eficiencia de su producto legislativo.

Este trabajo propuso fortalecer la profesionalización y permanencia de los técnicos, asesores y/o auxiliares legislativos o similares, para que ayuden con sus conocimientos y experiencia a los diputados, de manera responsable y profesional. Que es a través de la práctica profesional como lo han ido adquiriendo, pero que es debido a los periodos de tres años, y a que los diputados (as) traen consigo nuevo personal técnico de su confianza, que se interrumpe la experiencia formativa de los técnicos parlamentarios existentes.

El propósito último del servicio de carrera parlamentario es sustentar y potenciar la capacidad del Poder legislativo para bridar soluciones legislativas a los problemas sociales, y dar respuesta a las expectativas de la sociedad. Adicionalmente, la propuesta del sistema del servicio civil de carrera parlamentaria para los técnicos, asesores y/o auxiliares legislativos o similares pretende: a) aumentar la capacidad técnica de la 
asamblea legislativa; b) reducir las posibilidades de corrupción y/o cooptación por parte de actores políticos o actores privados sobre quienes trabajan al servicio de los legisladores; y c) mantener el seguimiento y control de las políticas implementadas por el gobierno estatal.

No obstante las limitantes que actualmente tiene la actividad legislativa para la profesionalización de los representantes populares, se enfoca principalmente a la actividad de los legisladores que tiene como objetivos: a) dar respuesta a las demandas de sus representados mediante la formulación y elaboración de iniciativas de ley, que conjugan los intereses de los legisladores y de sus partidos, con la respuesta a las demandas de sus representados (o electores); y b) la profesionalización se relaciona con la supervisión y control, mediante la rendición de cuentas de los funcionarios; así, se espera evitar el abuso de poder, e influir en las políticas, mediante la discusión pública y modificación de las propuestas. Y es precisamente para el cumplimiento de esta segunda tarea donde contribuye, en gran medida, la información generada por los especialistas del apoyo técnico.

\section{Este trabajo tuvo como limite el análisis acotado a quince ordenamientos en tanto:}

1) los congresos de los estados de Nayarit, San Luis Potosí y Baja California no cuentan con ordenamientos específicos;

2) el caso del congreso de Aguascalientes el ordenamiento fue abrogado;

3) si bien, las leyes orgánicas de los congresos de los estados de Yucatán, Tlaxcala, Puebla, Ciudad de México, Guerrero, Coahuila y Chihuahua anuncian la creación de ordenamientos específicos, éstos no se encuentran disponibles; y

4) el alcance de los aspectos regulados en los ordenamientos específicos estatales se vio rebasado en cuanto al tema de la definición operativa de servicio civil de carrera, ya que se identificó un séptimo proceso relativo a remuneraciones, sueldos, compensaciones salariales y prestaciones sociales.

Futuras líneas de investigación pudieran revisar con profundidad los ordenamientos específicos: los públicos y los enunciados en las leyes orgánicas, así como de las especificaciones (remuneraciones, seguridad, estabilidad, valores y principios) y singularidades (remoción, vocación de servicio, experiencia, etc.) de los criterios normados en cada uno de dichos ordenamientos, relacionándolos con la producción legislativa, con la percepción de la eficiencia de los colaboradores por parte de los 
legisladores, o bien con criterios específicos que contribuyan a una evaluación integral de éste servicio.

\section{LISTA DE REFERENCIAS}

Aguilar, L. (2003). La importancia del establecimiento del Servicio Público de Carrera en México. México: Universidad Iberoamericana. Conferencia presentada en el seminario sobre la Profesionalización del Servicio Público en México- Hacia la innovación y la democracia.

Campillo, M. (1999). Por un Congreso Inteligente. El Apoyo Técnico de staff en la Cámara de Diputados de México, 1988-1999. México: Tesis. Instituto tecnológico Autónomo de México.

Chávez, E. (2016). El Derecho Parlamentario Estatal Mexicano, Análisis y propuesta de reforma. México: Instituto de Investigaciones Jurídicas. UNAM.

Congreso de la Unión. (2008). El Servicio Civil de Carrera en el Ámbito Legislativo. Marco Teórico Conceptual, Marco Jurídico, Derecho Parlamentario y Opiniones especializadas. México: Congreso de la Unión.

De la Torre, E. S. (2019). El presidencialismo mexicano en su contexto histórico: instituciones y actores. Madrid: Tesis Doctoral. Universidad Complutense.

Escobar, S. (1997). La Asesoría Parlamentaria Externa en Chile. Washington, D.C.: OEA, Unidad para la Promoción de la Democracia y Centro de Estudios y Cooperación Legislativa.

Guerrero, J. P. (2000). Consideraciones sobre la instauración del servicio civil en México. México: Centro de Investigación y Docencia Económicas (CIDE).

IMCO. (2018). Informe Legislativo 2018. Ciudad de México: Instituto Nacional de la Competitividad.

INEGI. (2020). Censo Nacional de PoderesLegislativos Estatales 2020. Ciudad de México: INEGI.

Instituto Belisario Domínguez. (2018). Cuadernos de inducción: El Senado de la República y el trabajo legislativo. Serie para legisladores. México: Senado de la República.

Jaramillo, M. T. (2005). Responsabilidad Pública y Profesionalismo Parlamentario, como base fundamental de la reelección legislativa en nuestro país. Toluca: Tesis. Universidad Autónoma del Estado de México. 
Mondragón, A. (2014). Panorama del servicio parlamentario de carrera en los congresos locales en México: entre la simulación y el compromiso. Estado de México: Tesis. Universidad Autónoma del Estado de México.

Mora-Donatto, C. (2008). Urge rediseñar el servicio civil de carrera del congreso mexicano". Cuestiones Constitucionales. Número 19. Instituto de Investigaciones Jurídicas. UNAM. México. Cuestiones constitucionales, 174.

Moyado, S. (2006). La Importancia de la Gestión del Conocimiento en el Servicio Civil de Carrera de la Cámara de Senadores para el Aprendizaje Organizacional. Tesis Doctoral. México: Instituto de Estudios Superiores en Administración Pública.

Pardo, M. (2005). El servicio profesional de carrera en México: de la tradición al cambio. Foro Internacional, pp. 599-634.

Reyes Pastrana, J. (2020). Crónica Legislativa. Reseña Histórica del Poder Legislativo del Estado de México y sus Predecesores (1809-2021). . Toluca: Congreso del Estado de México.

SEDIA. (2018). El Servicio Civil de Carrera en el Ámbito Legislativo. Marco Teórico Conceptual, Marco Jurídico, Derecho Comparado y Opiniones Especializadas. México: Dirección de Investigación de Investigación y Análisis y la Subdirección de Análisis de Política Interior.

Siegel, S., \& Castellan, J. (2007). Estadística no paramétrica, aplicada a las ciencias de la conducta. México: Trillas.

Siegel,, S., \& Castellan, J. (2004). Estadística no paramétrica: aplicada a las ciencias de la conducta. México: Trillas.

Somma, A. (2014). Introducción al Derecho Comparado. Madrid: Universidad Carlos III.

Valencia, L. (2018). Representación parlamentaria, ciudadanía y rendición de cuentas vertical. Apuntes electorales, pp. 61-96. 